\title{
Elemental and configural olfactory coding by antennal lobe neurons of the honeybee (Apis mellifera)
}

\author{
Anneke Meyer • C. Giovanni Galizia
}

Received: 23 August 2011/Revised: 24 October 2011/Accepted: 26 October 2011/Published online: 15 November 2011

(C) The Author(s) 2011. This article is published with open access at Springerlink.com

\begin{abstract}
When smelling an odorant mixture, olfactory systems can be analytical (i.e. extract information about the mixture elements) or synthetic (i.e. creating a configural percept of the mixture). Here, we studied elemental and configural mixture coding in olfactory neurons of the honeybee antennal lobe, local neurons in particular. We conducted intracellular recordings and stimulated with monomolecular odorants and their coherent or incoherent binary mixtures to reproduce a temporally dynamic environment. We found that about half of the neurons responded as 'elemental neurons', i.e. responses evoked by mixtures reflected the underlying feature information from one of the components. The other half responded as 'configural neurons', i.e. responses to mixtures were clearly different from responses to their single components. Elemental neurons divided in late responders (above $60 \mathrm{~ms}$ ) and early responder neurons (below $60 \mathrm{~ms}$ ), whereas responses of configural coding neurons concentrated in-between these divisions. Latencies of neurons with configural responses express a tendency to be faster for coherent stimuli which implies employment in different processing circuits.
\end{abstract}

Keywords Hetero-glomerular local interneuron . Electroantennogram · Intracellular recording · Morphology $\cdot$ Mixture interaction

Electronic supplementary material The online version of this article (doi:10.1007/s00359-011-0696-8) contains supplementary material, which is available to authorized users.

A. Meyer · C. G. Galizia ( $\square)$

Department of Biology, University of Konstanz,

Constance, Germany

e-mail: giovanni.galizia@uni-konstanz.com

\author{
Abbreviations \\ AL Antennal lobe \\ EAG Electroantennogram \\ LN Local neuron \\ PN Projection neuron \\ ORN Olfactory receptor neuron
}

\section{Introduction}

Olfactory systems need to extract the biologically relevant information from chemically and temporally complex stimuli in a turbulent environment. This task is further complicated by the fact that in some incidences the important information might be carried by single compounds and in others by mixtures (for review see Lei and Vickers 2008). Accordingly, the olfactory system has to process configural information of the mixture as a whole (sometimes referred to as synthetic coding), but at the same time preserve elemental information about single compounds (sometimes referred to as analytical coding).

Olfactory guided behavior, which relies on efficient olfactory coding, is prominent amongst insects. Their comparably simple nervous system makes them excellent models to study mechanisms of the evolutionarily preserved olfactory system (Ache and Young 2005; Sato and Touhara 2009). The honeybee, Apis mellifera, has the ability to differentiate many odors, and is therefore well suited to study coding of complex odorant compositions (Menzel et al. 1996; Galizia and Menzel 2001). Importantly, in behavioral experiments bees are able to recognize a mixture of two odorants as a new odor ('configural coding'), but also to extract the information about the two odor components ('elemental coding') (Deisig et al. 2001, 2002, 2003). Thus, 
the olfactory system of the bee should allow for both, configural and elemental elements to be processed.

Configural coding is achieved in the primary olfactory area, the antennal lobe (AL). The AL consists of functional subunits with high synaptic density, the glomeruli. In each glomerulus three classes of neurons synapse onto each other: olfactory receptor neurons (ORN), projection neurons (PNs), and local interneurons (LNs). The AL network, build by these three types of neurons, is thought to reformat the input signal such that discernibility of stimuli is increased (Galizia 2008). ORNs detect odorant molecules at the antenna and form the input level of the AL. Each glomerulus receives sensory input from one type of ORN, which in honeybees branch throughout the superficial layer of the glomerular 'cap' (Pareto 1972). Optophysiological measurements of AL input in different insects indicate that it follows rules of elemental summation (Tabor et al. 2004; Deisig et al. 2006; Silbering and Galizia 2007).

PNs form the AL output and send their axons from the AL to higher processing areas (Mobbs 1982). In comparison to the input signal, representation of mixtures is more configural in PNs (Tabor et al. 2004; Silbering and Galizia 2007; Silbering et al. 2008; Deisig et al. 2010).

LNs branch exclusively within the AL and interconnect glomeruli. They are suggested mediators of linear and nonlinear transformations between AL input and output (Sun et al. 1993; Ng et al. 2002; Sachse and Galizia 2003; Sachse et al. 2006; Bhandawat et al. 2007; Olsen and Wilson 2008). In Hymenoptera, the honeybee in particular, two main morphological groups are distinguished: homo LNs uniformly innervate many glomeruli, and hetero-LNs innervate one glomerulus densely and several sparsely. Neurons of both groups can interconnect the cap and the central "core" within one glomerulus (Fonta et al. 1993). Similar to findings in moth, $\mathrm{LN}$ response latencies in the honeybee were found to be shorter than those of PNs suggesting that signal transfer from ORNs to PNs is mediated via LNs (Christensen et al. 1993; Krofczik et al. 2009). Furthermore, the presence of reciprocal synapses (Gascuel and Masson 1991) suggests a complex synaptic layout including both, LN mediated and direct ORN-PN signal transduction.

In the present study, we investigated in how far elemental odorant information is preserved in individual neurons. Further, we wondered if configural and elemental coding strategies can be attributed to morphologically different neurons. We approached these questions conducting intracellular recordings and morphological reconstructions from single AL neurons (LNs, as well as PNs) of the honeybee. Single cell recordings were combined with electroantennogram (EAG) recordings, in order to establish a precise temporal reference frame. To simulate properties of a turbulent environment, we created coherent or incoherent binary mixtures. In a coherent mixture, odorant components are delivered in synchrony, while in an incoherent mixture the components are delivered asynchronously.

\section{Materials and methods}

\section{Animal preparation}

Worker honeybees (Apis mellifera) were caught at the entrance of the hive or at a feeder, immobilized by cooling, and mounted in custom-made Plexiglas holders. The bees were allowed to acclimate to the new environment for 1-6 $\mathrm{h}$ before the experiment started.

Antennae were immobilized with Eicosane (melting point $37^{\circ} \mathrm{C}$; Sigma-Aldrich Chemie $\mathrm{GmbH}$, Germany) whilst head and mandibles were immobilized with Deiberit 502 (melting point $60^{\circ} \mathrm{C}$; Boehme-Schoeps, Germany). To reduce brain movements the esophagus was detached from its muscles. The head capsule was opened between the median ocellus and the base of the antennae. Glands and tracheal sheaths were removed carefully. The exposed brain was kept moist during recordings by dribbling saline onto it if necessary (in mM: $130 \mathrm{NaCl}, 6 \mathrm{KCl}, 4 \mathrm{MgCl}_{2}, 5$ $\mathrm{CaCl}_{2}, 10$ Hepes, 25 D-Glucose, 160 sucrose, $\mathrm{pH}$ 6.7, 500 $\operatorname{mosm} / \mathrm{L}$ ).

\section{Odorants}

Odorant identity (Krofczik et al. 2009) as well as concentration (Christensen et al. 1993; Stopfer et al. 2003) impact the response onset measured in electroantennogram recordings (EAG) as well as the latency of individual neurons (Junek et al. 2010). We chose two monomolecular odorants (1-octanol and 2-heptanone) that naturally occur in the honeybee's environment as both, components in floral mixtures (Omata et al. 1990; Tollsten and Knudsen 1992; Baraldi et al. 1999) and pheromones (Balderrama et al. 1996, 2002). We presented the single components alone, their coherent mixture (both components with synchronized odorant onset), and their incoherent mixtures (both components with odorant onsets shifted with respect to each other). In doing so, we created a controlled recreation of the elements in a dynamic odorant environment. In addition, comparing absolute response latencies can then be used to identify the odorant to which a particular neuron responds in a mixture, and thus to differentiate between 'elemental' and 'configural' coding (see below).

Stimulation paradigm

A custom-built olfactometer, similar to a previously published model (Galizia et al. 1997) was used for stimulation. Stimulus delivery was controlled by TTL pulses triggered 


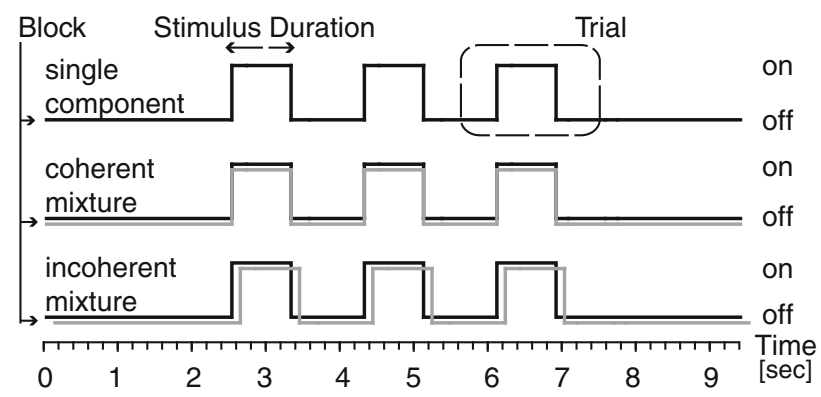

Fig. 1 Stimulus protocol. Stimulus duration was $800 \mathrm{~ms}$. The intertrial interval was $1,800 \mathrm{~ms}$. Each stimulus repetition was considered as one trial. One block consisted of three trials with identical stimulation. The onset delay for odorants in incoherent mixture blocks was $50 \mathrm{~ms}$

by the recording software, Clampex (AxonInstruments Inc., USA). Odorant flow through individual channels of the olfactometer was calibrated using a photoionization detector (Aurora Scientific Inc., Canada). The primary odorants 1-octanol and 2-heptanone, diluted in mineral oil were used for stimulation. Odorant concentration was 1:200 for 1-octanol and 1:100 for 2-heptanone. The effect of odorant concentration on response latency was tested with the following dilutions for 1-octanol and 2-heptanone, respectively: 1:200 and 1:100, 1:100 and 1:200, and 1:100 and 1:100. Airborne stimuli were delivered in a constant stream of clean air $(1.2 \mathrm{~m} / \mathrm{s})$ that was directed to both antennae. Stimulus duration was $800 \mathrm{~ms}$ at an inter-trial interval of $1,800 \mathrm{~ms}$. Three trials using identical stimuli followed each other in immediate succession and constituted one stimulus block. An interval of 5,000 ms separated two blocks from each other (Fig. 1). A completed recording consisted of the presentation of five stimulus blocks and two control blocks: each of the two primary odorants, their coherent mixture, their two incoherent mixtures, a control of mineral oil and a control of pure air. To an incoherent mixture the onset of the second odorant was delayed by $50 \mathrm{~ms}$ with respect to the first odorant. The sequence between presentations of different blocks was pseudo-randomized.

\section{Electrophysiology}

Three types of electrophysiological experiments were performed: electroantennogram (EAG) recordings, intracellular recordings from single AL neurons, and parallel recordings of both of these. EAG recordings served to validate the stimulus apparatus and to analyze odorant concentration-specific effects in ORNs. Intracellular recordings were used to investigate the role of single $\mathrm{AL}$ neurons within the AL network. Parallel recordings with both methods allowed measuring the response onset of single AL neurons with respect to a sensory time reference.
Glass electrodes were pulled from borosilicate capillaries (GC150F-10, Clark electronic instruments, UK) using a horizontal Puller (P-97, Sutter Instruments Co.,USA). Sharp electrodes $(100-250 \mathrm{M} \Omega)$ used for intracellular recordings were tip-filled with fixable fluorescent dye (4\% Alexa 488 hydrazid in $0.2 \mathrm{M} \mathrm{KCl}, 4 \%$ Micro Ruby in $0.2 \mathrm{M} \mathrm{K}$-Acetate or $3 \%$ Lucifer Yellow in $0.1 \% \mathrm{LiCl}$ ). Blunt electrodes (5-20 M $\Omega)$ used for EAG recordings were filled with $0.2 \mathrm{M}$ $\mathrm{NaCl}$.

The sharp electrodes were placed on the AL and gradually advanced employing a micro-manipulator (Kleindiek Nanotechnik, Germany) until a cell was impaled. For EAG recordings, a blunt electrode was placed on the antenna tip using a second micro-manipulator (Brinkmann Instrumentenbau, Germany). In cases where both signals were recorded in parallel, the EAG was always taken from the antenna ipsilateral to the AL recorded from. A common reference electrode was placed through a small incision in between the lower ocelli.

Recordings were performed in current-clamp mode, using an Axoclamp 2B Amplifier (gain 10, AxonInstruments Inc., SA). EAG signals were additionally amplified by means of a custom-built external amplifier (gain 10). A 50-Hz filter (Hum-Bug, Quest Scientific, Canada) removed line hum. Data were digitized using the Axon Interface, DigiData 1200B (AxonInstruments Inc., USA) and stored on hard-drive using Clampex 8.2 (AxonInstruments Inc., USA).

\section{Morphology}

After an intracellular recording was finished successfully, the dye loaded in the tip of the sharp electrode (see above) was iontophoretically expelled, with the polarity of the current pulses ( $0.2 \mathrm{~s}$ width, $2 \mathrm{~Hz}, 1-4 \mathrm{nA})$ chosen according to the dye's charge.

Subsequently, in order to visualize all glomeruli we counterstained the sensory tracts of the penetrated AL with Neurobiotin (Vector Laboratories Inc., USA). For this purpose, the cuticle previously removed from the head capsule was now repositioned and closed carefully with eicosan. The ipsilateral antenna was brought in an upright position and surrounded by a basin made from vaseline that was filled with $2 \%$ neurobiotin (in aq.dest.). The antenna was cut at the scapus and the neurobiotin was given $2-3 \mathrm{~h}$ to be taken up by the antennal nerve stump.

For morphological preparations, brains were removed and fixed in $4 \%$ paraformaldehyde for $3 \mathrm{~h}$ at RT, or overnight at $4{ }^{\circ} \mathrm{C}$. Subsequently, preparations were washed 3 times for 10,30 , and $45 \mathrm{~min}$ in phosphate buffered solution and incubated in $0.5 \%$ avidin-coupled fluorescent dye to visualize the neurobiotin in the ORNs (either AMCA-avidin, or cy3, depending on the single-cell 
marker) for at least $5 \mathrm{~h}$. Brains were then washed again 3 times for 15, 30 and $45 \mathrm{~min}$, dehydrated in an ascending ethanol series, cleared for $20 \mathrm{~min}$ in xylol and finally embedded in DPX mounting medium (Fluka, SigmaAldrich Chemie GmbH). To visualize staining results, confocal image stacks were taken with a Zeiss LSM 510 Meta confocal microscope (Carl Zeiss AG, Germany).

Data analysis

Sharp recordings were filtered off-line $(10 \mathrm{kHz}$ lowpass cutoff). Spikes were detected, using custom-written routines based on the open source $\mathrm{R}$ packages SpikeOMatic (Pouzat et al. 2004) and STAR (Pouzat and Chaffiol 2009). To determine firing rate and response latencies, algorithms provided by the open source Matlab toolbox FIND (http:// find.bccn.uni-freiburg.de) were employed. Analysis of single cell data was chosen so as to maximize comparability to related work (Krofczik et al. 2009). Image processing of confocal stacks and reconstruction of cell morphology were achieved using AMIRA 5.1 software (Mercury Computer Systems, Germany). For analysis of EAG recordings, custom-written routines in $\mathrm{R}$ (http://www. R-project.org) were used.

\section{Temporal electroantennogram analysis}

EAG recordings were filtered off-line (100 Hz low-pass), and averaged over repeated trials. Response onset was defined as the relative maximum preceding the steepest negative slope of the potential drop which demarcated an odorant response. This point was found to be least affected by temporal displacement attributable to response amplitude and, hence, evaluated as most reliable.

\section{Response latency analysis}

Using sharp electrodes intracellularly, we recorded from a total of 21 cells in the antennal lobe. Neurons differed greatly in their physiological properties: some had low background activity, and responded to odors with single or few spikes (Fig. 2b, right), some responded with clearly increased firing rate (excited, Fig. 2b, left), others with a drop in spike rate (inhibited, Fig. 2b, middle). In neurons that yielded a good morphological staining it was possible to identify their morphology as PNs or LNs, in all other cases this was not possible, due to the 'blind' nature of our recording: we refer to the presumably mixed population of recorded LNs and $\mathrm{PNs}$ as $\mathrm{AL}$ neurons. We assume that no

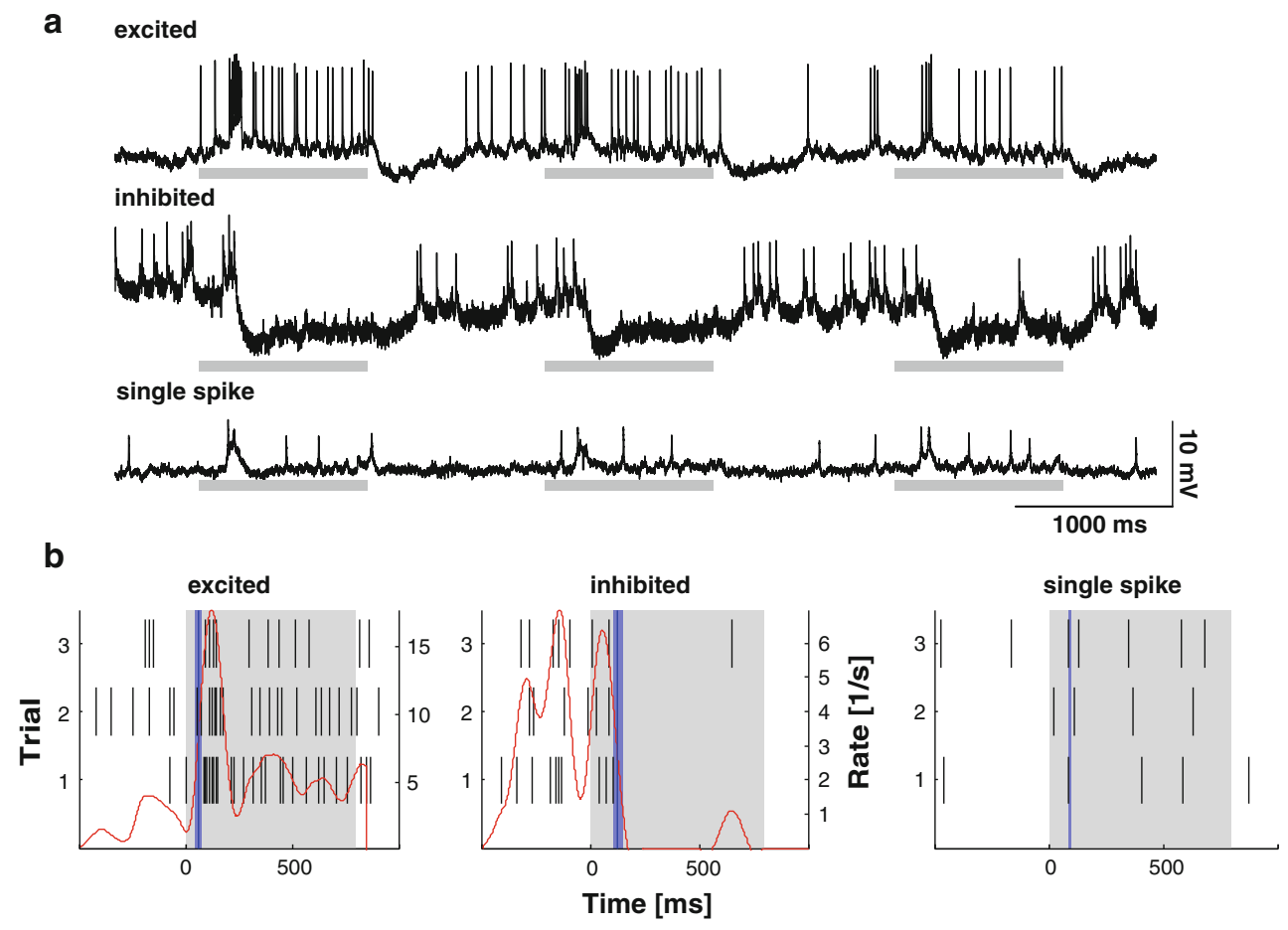

Fig. 2 Estimation methods of response latency was chosen with respect to response patterns. a Exemplary traces of neurons with different response patterns: excitation (top), inhibition (middle), or few spikes on a depolarization (bottom). b Estimation of response latency. Spiking activities shown for three repeated trials under identical stimulation. Superimposed red traces indicate the response rate function. Blue lines mark the estimated response onset and blue bars indicate their across trial variability. Response onset for excitation is defined as the point of steepest rising in the rate function after stimulus onset (left). Response onset for inhibition is defined as the point of steepest falling (middle). Response onset for neurons with continuously sparse firing is not well captured by a rate function and best characterized by the peak time of a single spike after depolarization onset (right). Grey bars indicate stimulus delivery time 
ORNs were stained, because the small axon terminals of these neurons make it unlikely that we impaled them with our sharp electrode. In our preparations we never found stainings of neurites directed towards the antennal nerve. We judged a cell as responding to a stimulus, if repeated stimulation elicited similar modulation in the neuron's firing pattern. Absolute latency, that is the mean latency across trials, and relative latencies, that is trial-to-trial differences in latency, were calculated with one of three methods (1-3). The method was chosen based on the respective firing pattern (Fig. 2).

1. Latencies of cells that responded to stimulation with an increased firing-rate were estimated based on the derivative of the trial-aligned firing rate as described elsewhere (Meier et al. 2008). This method processes the data in four successive steps. First, the derivative of each single trial spike train of a given cell was estimated by convolving with an asymmetric Savitzky-Golay filter (Savitzky and Golay 1964) (polynomial order 2, 300 ms width, Welch windowed). Second, all single trial-derivatives were optimally aligned, finding the greatest possible pair-wise cross correlation (Nawrot et al. 2003). The resulting timeshifts correspond to each trial's relative latency. Their standard deviation $\sigma$ gives a measure for the acrosstrial latency variability. Third, the single trial spike trains are temporally aligned by shifting each by its individual relative latency. Fourth, the aligned spike trains were merged into one train, representing the cells activity pooled over trials. The convolution of this merged spike train with the same asymmetric Savitzky-Golay filter gave an estimate about the derivative of the cell's absolute firing rate, based on which the absolute latency within a given block of stimulation was determined. The stimulus-specific absolute latency was defined as that point in time where the slope of the firing rate is steepest, that is the derivative's maximum.

2. Latencies of cells that responded to stimulation with a decrease in firing rate were estimated with an approach nearly identical to 1), but instead of the steepest rising slope, the steepest falling slope of the absolute firing rate was defined as response onset.

3. Latencies of cells that had very low spontaneous activity and responded to stimulation with a membrane depolarization ridden by one or few single spikes were estimated based on spike peak time rather than rate. The membrane depolarization in these cells was taken as indicative for an apparent response. The response latency was defined as the peak time of the first spike riding such a depolarization.

\section{Single cell morphology}

Confocal image stacks were processed using Amira 5.1 software (Visage Imaging GmbH, Germany). All preparations were carefully inspected for complete stainings: specimens with irregular labeling, abruptly ending neurites or strong fluorescent background were excluded from further morphological analysis. However we did include preparations in which the neuron was presumably incompletely filled but sufficient to transfer valuable information. Incomplete stainings can never be excluded in intracellular dye fills, for example due to extremely small neurite diameter and consequently compromised distribution of fluorescent dye. We searched very carefully for stained axons leaving the $\mathrm{AL}$ in order to differentiate between LNs and PNs. Neurons were reconstructed using the filament editor without further estimation of neurite diameter. Location and size of single glomeruli were registered by interactive segmentation based on OSN mass fills. Glomerulus identity was determined by visual inspection and comparison with the morphological atlas of the honeybee (Galizia et al. 1999, http://neuro.uni-konstanz.de/honeybeeALatlas). To compare innervation patterns with spatial patterns of AL activity in response to the stimuli applied, the physiological atlas of the honeybee (http://neuro.uni-konstanz.de) was consulted.

\section{Results}

Latency shifts in receptor neurons are inherited by antennal lobe neurons

We chose two odorants and analyzed latency difference between them at two different concentrations. At the level of the compound olfactory receptor neuron response as measured with EAGs, we found that response latency decreases with increasing concentration (two-way Anova, $F_{\text {concentration }}<0.000$; Tukey test, $p_{\text {concentration }}<0.000$; mean difference $=22.646 \mathrm{~ms}$ ) irrespective of the odorant used ( $p_{\text {odorant }}=0.7 ; p_{\text {interaction }}=0.9$ ).

In order to investigate how ORN latency shift transfers to the AL network we conducted simultaneous intracellular and EAG recordings (Fig. 3a). The odorant at lower concentration evoked a smaller EAG amplitude (Fig. 3b) as well as a weaker firing rate in phasic-tonic AL neurons (Fig. 3c). As for EAGs, the latency in $\mathrm{AL}$ neurons decreased with increasing odorant concentration (one-sided, paired $t$ test, $p=$ 0.048). Indeed, the difference between mean response onsets of EAGs and AL neurons was identical for both stimuli (37 ms; Fig. 3d). Thus, concentration-dependent latency shifts originate in ORN activity and transfer directly to AL 

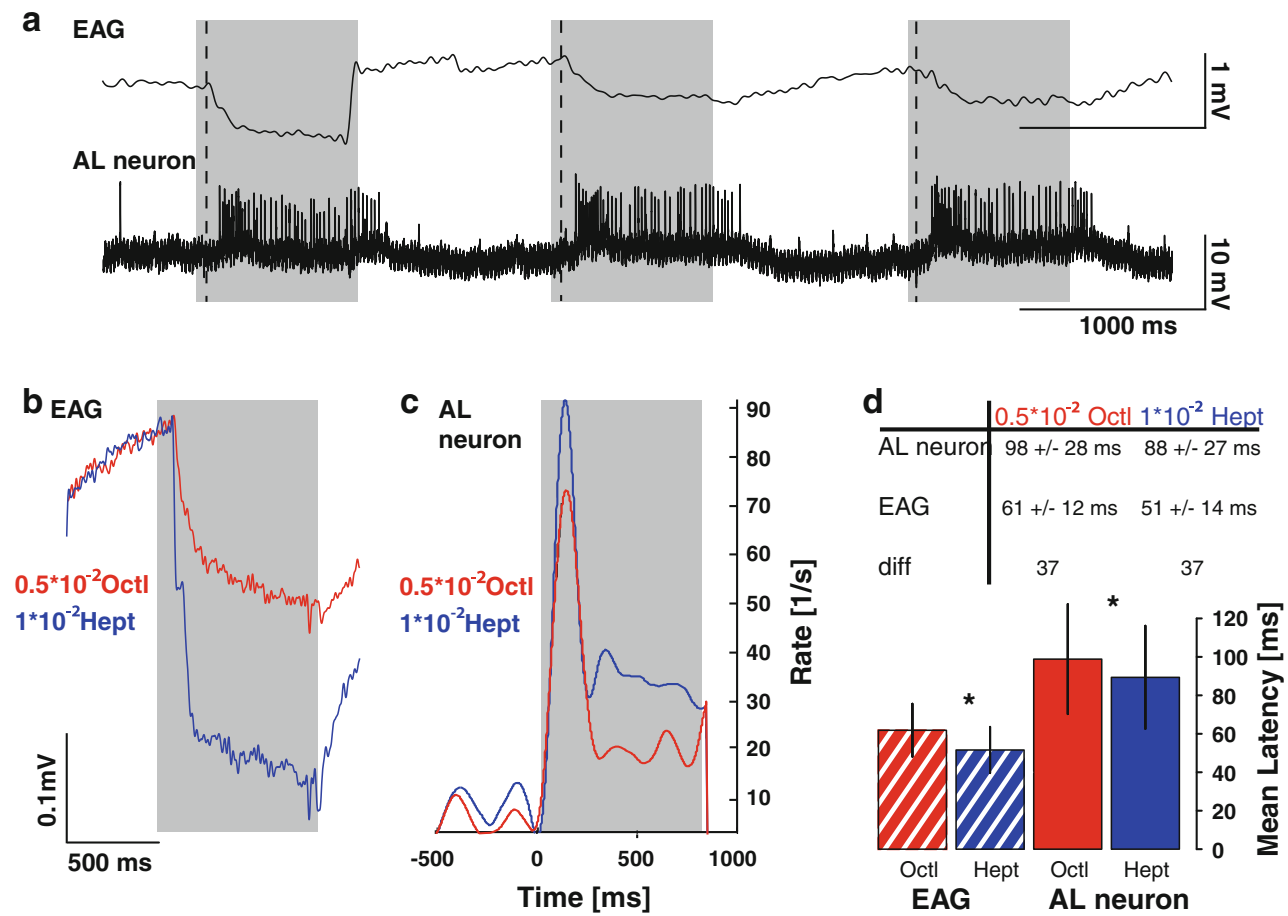

Fig. 3 AL neurons inherit a concentration-dependent latency shift from peripheral neurons. a Exemplary traces of simultaneous EAG and single-cell recording. Grey bars indicate stimulus delivery time. Dotted lines indicate the time of stimulus detection on the antennae, measured by EAG (upper trace). b EAG amplitude differs for different stimuli. EAG in response to lower concentrated 1-octanol is depicted in red. EAG in response to higher concentrated 2-heptanone is depicted in blue. Traces shown are averaged $(n=11)$. c AL neurons

neurons. Accordingly, odorant concentration can be used to create stimuli with distinct, characteristic latency shifts. This phenomenon offers a tool to identify a response evoking component from a mixture, within single AL neurons.

Simultaneous recording of AL neurons and EAG also allowed us to report the response latency of single neurons more accurately. Timing of olfactory stimuli is always constrained by laboratory conditions, like air turbulences or distance between odorant source and receiver (Vetter et al. 2006). While easy to control within one lab, these constraints are difficult to standardize between different laboratories. Comparability of data between laboratories is increased when latencies are estimated with respect to a reference derived from the animal rather than the stimulation machinery. The EAG reliably reflects stimulus arrival at the antennae. Hence we used the mean EAG response onset as a reference time point to estimate single AL neuron latencies.

Both, elemental and configural coding occur in antennal lobe neurons

In a natural environment, components of an odorant mixture might impinge on the antenna simultaneously (as in a mean firing rate differs for different stimuli. Color code as in $\mathbf{b}$. Rate functions are aligned and averaged over all neurons which responded with a phasic-tonic pattern $(n=12)$. (d) Response latencies of EAG and AL neuron recordings differ significantly for different stimuli, but keep the same relative time shift $(37 \mathrm{~ms})$. Color code as in b, c. Bars illustrating mean latency in EAGs are stripy. Bars illustrating mean latencies from single cell measurements are colored solid. Same animals as in $\mathbf{b}$, $\mathbf{c}$; error bars give standard deviations

mixture from one source), or separate in time (as when they originate from different sources). An olfactory system that can differentiate between these two conditions might be able to select whether to process a mixture in a configural way (i.e., the mixture being more than the summation of its components), or in an elemental way (i.e., with access to the components' identity). To study this question, we presented single odorants and their coherent and incoherent mixtures. We asked whether single AL neurons can be attributed to elemental or configural processing, and whether their coding strategies are related to the cell's own response latencies.

About half of all recorded AL neurons responded to the mixture in the same way as to one of its components $(n=$ 12, 'elemental neurons'). The remaining cells $(n=9$, 'configural neurons') responded to the mixtures as to a different odorant.

'Elemental neurons' fell in two selectivity groups: element selective and element dominant.

The first group ( $n=7$, element selective) responded to the mixture as to one of its components, its dominant (D) odorant. Response latency to the coherent mixture, and the incoherent mixture in which odorant $\mathrm{D}$ was presented 
a

$\mathbf{S}$

.

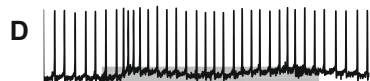

SD

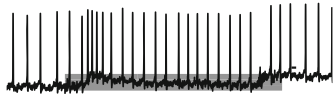

S-D

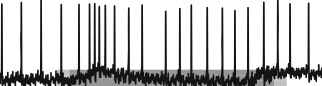

D-S

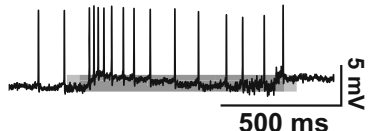

C

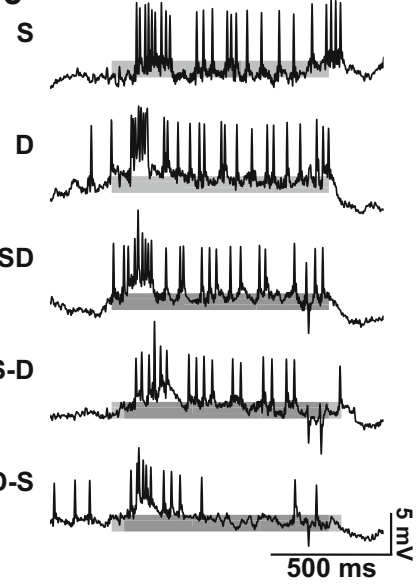

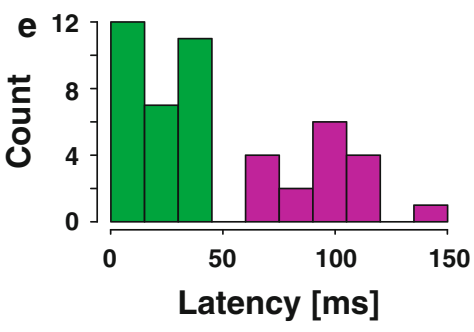

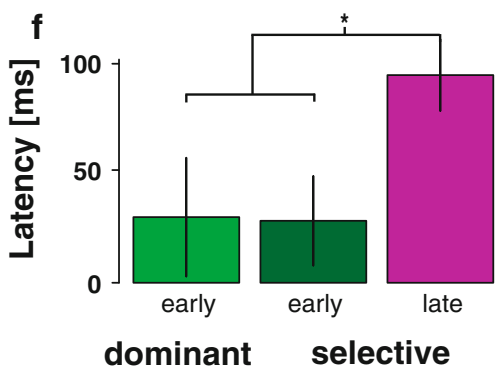

g

\begin{tabular}{|c|c|c|c|}
\hline & $\begin{array}{l}\text { early } \\
\text { dominant s }\end{array}$ & $\begin{array}{l}\text { earl } \\
\text { selec }\end{array}$ & $\begin{array}{l}\text { late } \\
\text { selective }\end{array}$ \\
\hline mean [ms] & 31 & 28 & 93 \\
\hline $\mathrm{sd}[\mathrm{ms}]$ & 28 & 20 & 16 \\
\hline
\end{tabular}

dominant

selective

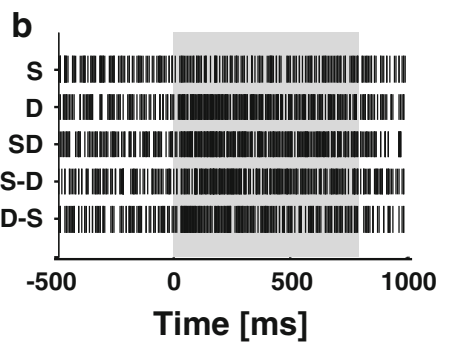

s $\cdot\||\||||||||||||||||||||||||||||||||||||||||||||||||||||||||||||$

D - ||||| ||||||||||||||||||||||||||||||||||||||||||||||||||

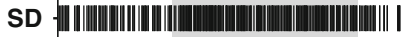

S-D $\cdot\||\||||||||||||||||||||||||||||||||||||||||||||||||||||||$

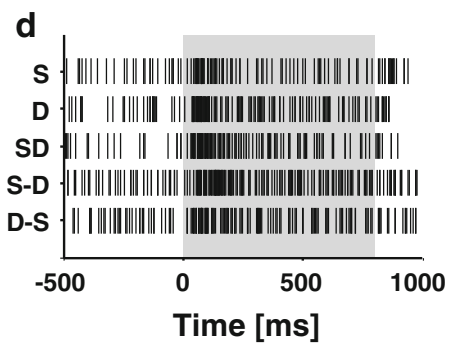

preference of odorant $D$. d Merged spike trains comprising all element-dominant neurons $(n=7)$. Note that subordinate $(S)$ as well as dominant $(D)$ odorant evoke a response. e Irrespective of selectivity, neurons' latencies cluster into an early (green) and a late (magenta) group. f Based on the combination of latency and selectivity, three neuron groups become apparent. Element-dominant neurons always respond early (light green), element-selective neurons distribute in an early (dark green) and a late group (magenta), with significant different latencies (paired $t$ test, one-sided, $p=0.001$ ). $\mathbf{g}$ Table of mean cell latencies sorted according to group

cluster, while element-selective neurons distributed into both clusters. Based on the combination of selectivity and latency, we end up with three subgroups of 'elemental neurons': element-dominant with early responses $(n=5)$, element-selective with early responses $(n=4)$ and elementselective with late responses $(n=3)$ (Fig. 4f, g).

Responses of 'configural neurons' were more diverse, both in terms of selectivity and latency. One cell responded only to coherent stimuli (Fig. 5a), some to mixtures only $(n=2)$, but most cells responded to mixtures as well as single compounds ( $n=6$, Fig. 5b). Response latencies of 'configural neurons' scattered broadly around $60 \mathrm{~ms}$ and thus concentrated exactly between the groups of 'elemental neurons' with early and late responses (Fig. 5c, d). Unlike for 'elemental neurons', latencies within one 'configural neuron' could be short for one stimulus and long for another one. Fastest responses were, in the mean, evoked by single compounds $(62 \pm 26 \mathrm{~ms})$ and slowest by ment-dominant neurons fell into the early responders 
a
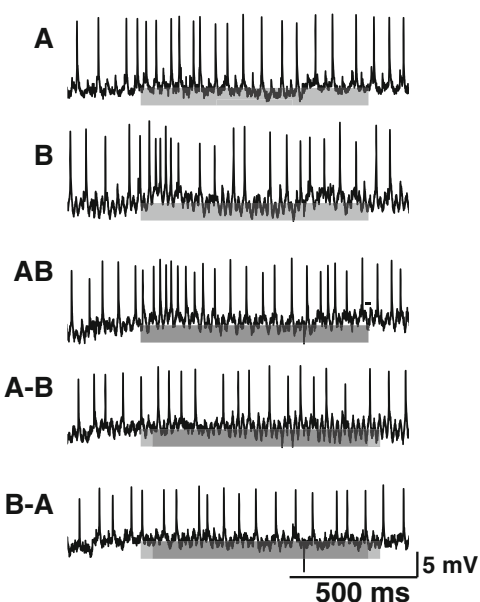

b

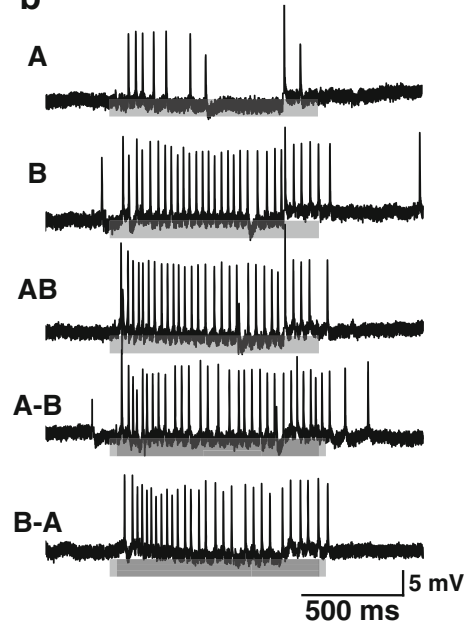

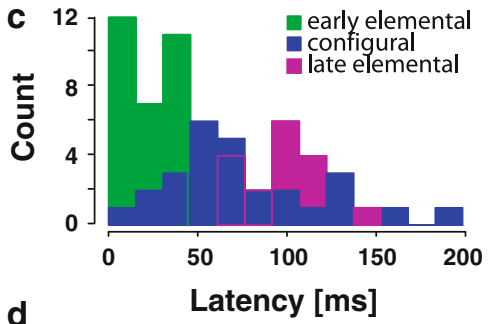

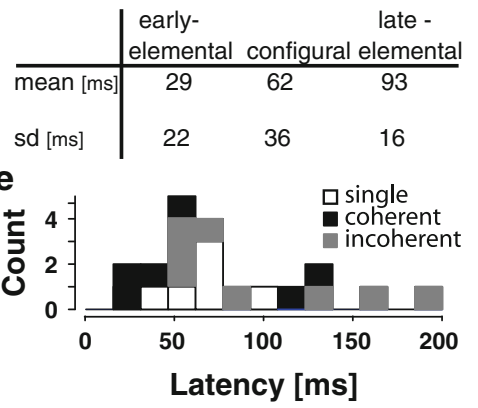

Fig. 5 'Configural neurons' and distribution of latencies with respect to 'elemental neurons'. a Responses to coherent stimuli only [Latencies (ms): $B$ 94, $A B$ 132). Grey bars indicate stimulus delivery time. b Example of an AL neuron which responded to single compounds and coded mixtures configurally $[n=6$, latencies of the individual neuron (ms): $A$ 62, $B$ 49, ab 61, $A-B$ 67, $B-A$ 87]. c Histogram of latencies from neurons with configural responses (blue), early (green) and late (magenta) neurons with elemental responses (green and magenta as in Fig. 4e). d Table of mean cell latencies sorted according to group. e Histogram resolving response latencies of 'configural neurons' to single compounds (white), coherent mixtures (black) and incoherent mixtures (grey)

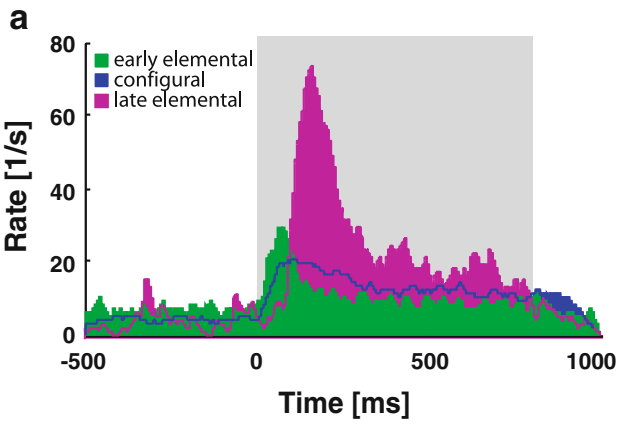

Fig. 6 Early responding AL neurons may impact late responses in AL neurons. Group averaged rate functions of early responding 'elemental neurons' (green trace, $n=9$ ), late responding 'elemental neurons' (magenta, $n=3$ ) and 'configural neurons' (blue trace, $n=6$ ). Grey shades indicate stimulus delivery time. The odorant-selective, late group comprised responses of excitation (a) and inhibition

incoherent mixtures ( $84 \pm 49 \mathrm{~ms}$, paired $t$ test, $p<0.05$; Fig. 5e).

Antennal lobe neurons are active sequentially

We analyzed response latencies in more detail by calculating population rate functions for each of the subgroups (Fig. 6). Early responses of 'elemental neurons' were either excited, or single spikes. We pooled these and estimated a common rate function. Late responses of 'elemental neurons' were either spike excited or inhibited responses,

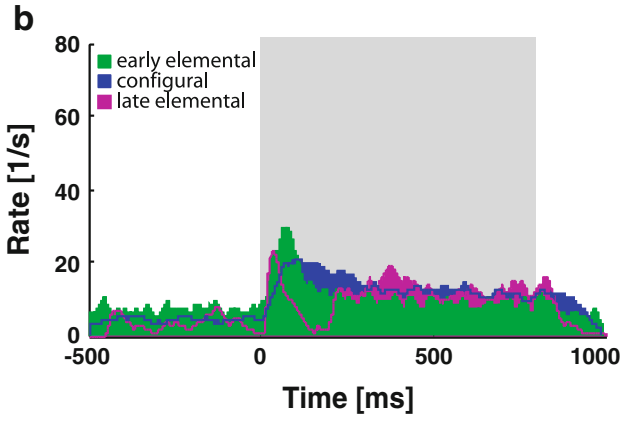

(b) which are treated separately. a Rate peaks of early neurons precede positive rate peaks of excited late AL neurons. b Rate peaks of early neurons also precede negative rate peaks of inhibited late neurons. Note the initial small activity boost which precedes the inhibited period

which we separated in two rate functions. Amongst the 'configural neurons' all types of responses were represented. We pooled all non-inhibited responses in a common rate function.

Superimposition of these rate functions illustrates that activity peaks of the three groups follow each other in immediate succession, suggesting that the corresponding cells might be arranged sequentially in a functional network. Early responding elemental AL neurons (green) precede the positive rate peak of excited (magenta; Fig. 6a), as well as the negative rate peak of inhibited late 
responding 'elemental neurons' (magenta; Fig. 6b). Interestingly, the rate function of inhibited late responding neurons appears to have a small activity boost, which relapses just before early responding neurons reach their maximal response frequency. While 'elemental neurons' have rather distinct activity peaks, 'configural neurons' appear more diverse, reflecting the scatter in latencies within and across this neuron group.

Hetero local neurons are involved in configural as well as elemental processing

What is the relationship between elemental and configural responses on one side, and the cell's morphology on the other? Using intracellular staining we obtained the morphology of one PN and three LNs. The PN fell into the element-selective, late response group. The LNs all had early mean responses. Two of the LN stainings were of sufficient quality to be reconstructed into a 3D skeleton model (Fig. 7). Both neurons responded to 2-heptanone and to its binary mixtures (Fig. 7a, h) and were hetero-LNs, i.e. with one densely innervated glomerulus ('main glomerulus') and several sparsely innervated glomeruli. One was an odorant-selective 'elemental neuron' (Fig. 7, top row) and the other a 'configural neuron' (Fig. 7, middle and bottom row). We asked whether their glomerular innervation pattern could explain their different response profiles. We identified the innervated glomeruli and compared these with the AL's spatial activity pattern evoked by 2-heptanone as published in the physiological atlas of the honeybee (http://neuro.uni-konstanz.de; c.p.: Fig. 7f). The densely innervated glomerulus of the 'elemental neuron' was one of the 2-heptanone responsive glomeruli (T1-29, Fig. $7 b, c)$. The neurites branched within the core of the glomerulus and reached out into an intermediate layer between cap and core. Counterstaining of ORNs showed that $\mathrm{LN}$ branches and ORN axons overlapped suggesting that a direct input from ORNs is possible (Fig. 7d, white arrows). The main glomerulus of the 'configural neurons', however, innervated a glomerulus that is not responsive to 2-heptanone (T1-19, c.p.: Fig. 7f, g). This neuron innervated several glomeruli sparsely, among which at least three that are weakly responsive to 2-heptanone (T3-18, T3-31, T3-52; Fig. 7i, j; see also movies in supplemental material). Sparse arborization fibers did not reach into the glomerular cap (Fig. 7k1, magenta arrows), which would suggest that this neuron did not receive input from ORNs. However, a careful reconstruction of the neuron and the glomerular cap based on counterstained ORNs showed that the sparsely arborizing neurites in fact distributed just between cap and core (Fig. 7k2, magenta-white arrows). Hence, from our data we cannot decide whether heteroLNs have, in their sparsely innervated glomeruli, direct, monosynaptic ORN input from the cap, poly-synaptic input through LNs and PNs from the core, or both.

While the response latency of the 'elemental neuron' to the dominant odorant and its mixtures was similar ( $36 \pm 2$ vs. $38 \pm 4 \mathrm{~ms}$ ), the 'configural neuron' clearly responded faster to the single compound than to the mixtures (18 \pm 1 vs. $49 \pm 11 \mathrm{~ms}$ ). This change in latency indicates the occurrence of both, mono- and poly-synaptic input to the configural processing $\mathrm{LN}$. We hypothesize that this neuron was embedded in two different processing circuits that were differentially activated depending on the sensory stimulus delivered.

\section{Discussion}

In the present study, we investigated how single neurons in the honeybee antennal lobe (AL) process odorant mixtures. More particularly, we wanted to know if it is possible to differentiate between 'elemental' and 'configural' neurons. We found that indeed, there are 'elemental' and 'configural' neurons, though the same neuron may fall into either category depending on the particular stimulus used. Furthermore, the fastest neurons have elemental responses, while neurons with configural response properties respond later (Fig. 6), suggesting that computing configural responses is more demanding to the network than computing elemental responses. At the same time, configural coding is not the end result of AL processing, as shown by even later responses of elemental neurons.

It should be noted that intracellular recordings in the honeybee antennal lobe only allow for short measurements (less than $10 \mathrm{~min}$ ), and therefore the sample size of stained neurons is small. Thus, some of the observations that we draw, in particular about the relationship between morphology and functional properties, need to be taken more as hypotheses than as proven facts. However, together with published reports from our colleagues (Flanagan and Mercer 1989; Fonta et al. 1993; Galizia and Kimmerle 2004; Krofczik et al. 2009), these data help understanding the intricate coding networks in the insect antennal lobe, in particular with respect to mixture coding.

In what situation would elemental coding on the one, and configural coding on the other hand be relevant for an organism? Natural odorants as they are produced by, e.g. flowers are usually complex blends of many chemical compounds. A pollinating bee will perceive this bouquet as an individual odor, much as we do the smell of coffee. In an other instance, the bouquet of two different flowers might mix in the air. Here, the olfactory system has the task to separate odor components, i.e. to analyze the elements of the mixture: 'it smells two different flowers' or 'it smells coffee and fresh baked bread'. In a turbulent environment 

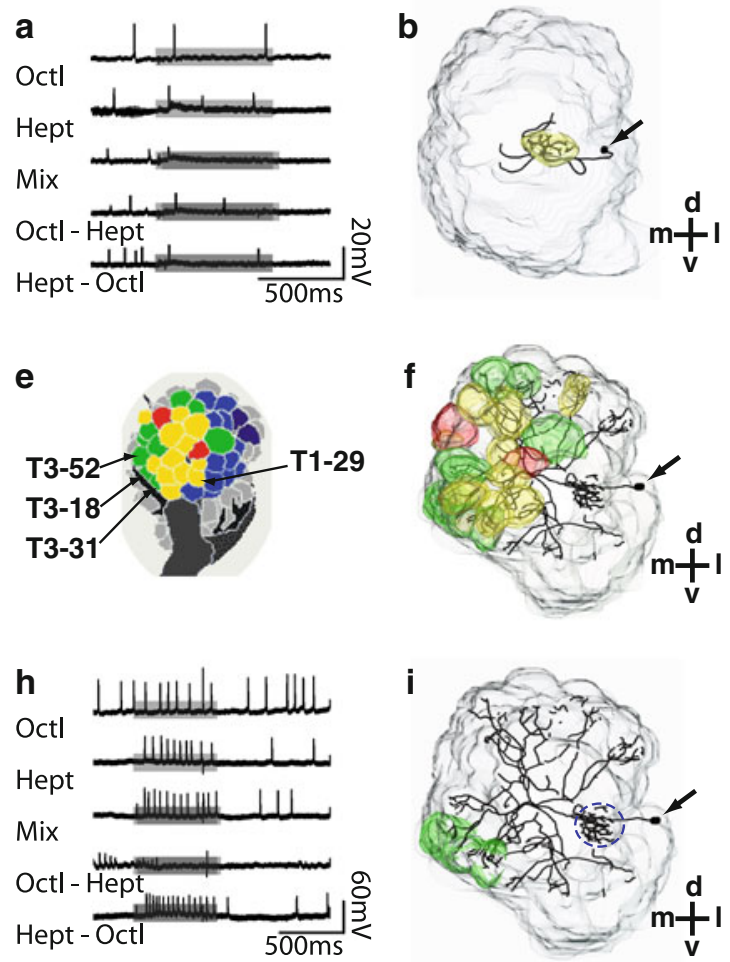

Fig. 7 Glomerular innervation patterns of hetero-LNs responding to 2-heptanone. a Electrophysiological recording from a hetero-LN exhibiting elemental coding of 2-heptanone. b Frontal view of a reconstruction of the neuron corresponding to the traces in $\mathbf{a}$. The highlighted glomerulus (T1-29) is known to be responsive to 2-heptanone and densely innervated. c Parasagittal view of the same hetero-LN as in b. d Confocal image illustrating the type of dense innervation observed in the neuron in $\mathbf{b}, \mathbf{c}$. ORN innervation is given in green, LN innervation in magenta. Note the overlapping innervation area (white arrows). e Schematic drawing of the AL illustrating in color code the involvement of single glomeruli in the response to 2-heptanone, as determined by calcium imaging with bath applied dye (cp. http://neuro.uni-konstanz.de/honeybeeALatlas for the physiological atlas of the honeybee). Arrows indicate glomeruli which are innervated by the neurons presented. f Color coded 2-heptanone response as determined in calcium imaging experiments, projected on a reconstruction of a hetero-LN exhibiting configural coding of

odorants from different sources frequently mix and we assume that the 'coffee' mixture will generally travel as a coherent mixture, while 'coffee and bread' will more likely generate incoherent mixtures. This hypothesis has been tested using moth sexual pheromone components: when the components were released from the same location, male moths were attracted by the mixture ('coherent' case, the moth recognized the mixture as an odor), while spatially separated locations did not attract the males ('incoherent' case, the moth did not behave as to a mixture, suggesting elemental coding (Andersson et al. 2011). In our study of mixture processing at the level of single neurons in the $\mathrm{AL}$ of the honeybee, we have therefore used two kind of mixture stimuli: coherent and incoherent mixtures.
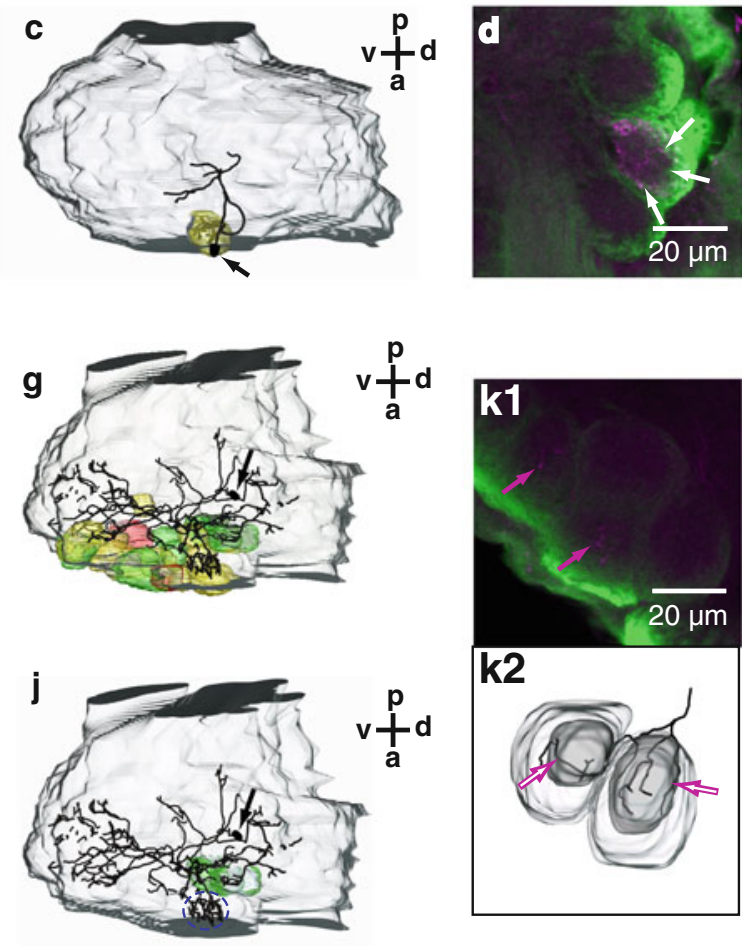

2-heptanone. Clearly the main glomerulus (T1-19) is not part of the stereotypic 2-heptanone response pattern. g Parasagittal view of $\mathbf{f}$. $\mathbf{h}$ Electrophysiological recording from the hetero-LN in $\mathbf{f}, \mathbf{g}$ exhibiting configural coding of 2-heptanone. i Frontal view of the same neuron as in $\mathbf{f}-\mathbf{h}$. The highlighted glomeruli (T3-52, T3-18, T3-31) are sparsely innervated by the depicted neuron and known to be responsive to 2-heptanone. Blue dotted line indicates the location of the densely innervated glomerulus (T1-19). $\mathbf{j}$ Parasagittal view of $\mathbf{i}$. $\mathbf{k} 1$ Confocal image illustrating the sparse innervation in $\mathbf{i}, \mathbf{j}$ of glomeruli in the core region. Sparse arbors seem not to overlap with ORNs (magenta arrows). k2 Reconstruction of glomerular cap and core as well as sparse arbors from $\mathbf{k}$, i. Note that neurites distribute just between cap and core (magenta-white arrows). $d$ dorsal, $v$ ventral, $m$ medial, $l$ lateral, $p$ posterior, $a$ anterior. $\mathbf{f}, \mathbf{g}, \mathbf{i}, \mathbf{j}$ are available as movies in supplemental material for better visualization. Arrows in $\mathbf{b}, \mathbf{c}, \mathbf{f}, \mathbf{g}, \mathbf{i}, \mathbf{j}$ indicate cell body

When looking at the response of a single neuron to the mixture $\mathrm{AB}$ of two odorants $\mathrm{A}$ and $\mathrm{B}$, it is not always a trivial task to characterize whether the neuron responded to $\mathrm{A}$ or to $\mathrm{B}$, or to the mixture. A neuron that does not respond to either $\mathrm{A}$ or $\mathrm{B}$, but responds to $\mathrm{AB}$, is clearly a 'configural neuron', selective to the mixture stimulus. But how about a neuron that responds both to $\mathrm{A}$ and to $\mathrm{B}$, and also to the mixture $A B$ ? Does it respond to a single one of the components, to both individually, or to the mixture? We made use of concentration-dependent latency shifts that are generated in the receptor neurons to address this question. We recorded EAG potentials in parallel to intracellular recordings, and could determine response latencies with great precision. We also titered odorant concentration in a 
way to generate distinct latencies for the two odorants A and B. Using the distinct latencies as a marker for stimulus identity, it was possible to infer for each neuron if a response was evoked by a mixture as a whole, or by one of the mixture compounds.

We found that half of the neurons responded to one of the compounds rather than the mixture: 'elemental neurons'. These neurons could be subdivided into elementselective neurons with early responses, element-dominant neurons with late responses, and element-selective neurons with late responses. Honeybee LNs have been shown to respond faster to odorant stimulation than PNs (Krofczik et al. 2009), which would suggest that the early responding neurons are LNs, the late responding PNs. Since our sample of stained neurons is small, in this work we do not differentiate among them, and lump them all as $\mathrm{AL}$ neurons.

The other half of the neurons gave 'configural' responses, i.e. their response patterns to the single components and to the mixtures differed, and in particular mixture responses did not reflect feature information of single compounds. Response latencies of 'configural neurons' varied considerably, but responses were often faster for coherent than for incoherent stimuli. This observation suggests that these neurons may be embedded in different processing circuits, depending on the stimulus components. This property may be used in context-dependent coding, whether the context is another stimulus or the physiological state of the animal.

Electrophysiological recordings have the advantage of high temporal resolution and thus allow to detect minute delays of responses within and between neurons. Previous studies have shown that LNs respond prior to PNs (Krofczik et al. 2009) and recordings in moth revealed LN subgroups with different latencies that allow for LN-LN interaction (Christensen et al. 1993). These findings suggest the existence of successively active neuron populations.

In agreement with previous data from the honeybee (Flanagan and Mercer 1989; Sun et al. 1993), we could not find latency clusters comparable to those shown for the moth. Our finding that 'configural neurons' had a tendency to respond later to incoherent (delayed stimulus onset of two compounds) than to coherent stimuli (single compounds and their temporally coherent mixture) suggests a less well ordered, but functionally more flexible ALarchitecture: these neurons may be recruited by different functional networks, depending on the network activity elicited by the stimulus.

We found another observation hinting towards non-linear processing mechanisms in the $\mathrm{AL}$, in neurons with late elemental responses: inhibited late responding neurons had a small activity boost, relapsing just before early responding neurons reached their maximal response frequency. It is inspiring to think that this finding could be explained by recurrent inhibition, as is known between granule and mitral cells in the mammalian olfactory bulb (for review see Urban and Arevian 2009).

Taken together, the data shown here and in other studies show that the AL is not a simple feed-forward relay station, but a dense and multi-layered neural network with recurrent connectivity.

Local neurons with asymmetric shape have been found in many insect species (Flanagan and Mercer 1989; Stocker et al. 1990; Christensen et al. 1993; Sun et al. 1993; Seki and Kanzaki 2008; Chou et al. 2010). Hetero-LNs as found in honeybees, with a single, densely innervated glomerulus and a limited number of sparsely innervated other glomeruli, have so far only been found in bees, and occasionally in other hymenoptera (Dacks et al. 2010). What is the specific function of these neurons? Based on their morphology, hetero-LNs have been proposed to be functionally polarized, with the main glomerulus being dendritic, and the sparsely innervated glomeruli being axonal (Galizia and Kimmerle 2004). This 'central-input' polarity is useful to shape inter-glomerular inhibition and elemental mixture processing, in particular given that inter-glomerular inhibition is dependent on the functional similarity of glomeruli (Linster et al. 2005). The neuron shown in Fig. 7a-c shows all the features of such a neuron, in particular given its innervation of glomerulus T1-29, which receives OSN input responding to 2-heptanone, an odorant that elicits response in this neuron. However, the neuron shown in Fig. 7d-k does not fit this pattern. This suggests that some hetero-LNs may have another polarity ('central-output'): dendritic innervation in the sparse glomeruli, and axonal in the dense glomerulus, as suggested from developmental studies where pruning in a dendrite-like fashion was observed in sparse but not dense arbors (Devaud and Masson 1999). A central-output hetero-LN may be suited to create configural response patterns, when its focal inhibitory action on a particular glomerulus is based on a distributed input from a defined glomerular assembly. However, the data also allows a third possibility. HeteroLNs might not have a fixed polarity (and form two groups, i.e. 'central-input' and 'central-output'), but they might in fact act either way depending on the activity pattern in the antennal lobe. Dendrodendritic interactions have been shown in olfactory granule cells in the vertebrate olfactory bulb (Shepherd et al. 2007), and reciprocal synapses abound in insect antennal lobes, as shown in cockroach (Malun 1991; Distler et al. 1998) and bees (Gascuel and Masson, 1991). Multiple spike heights have often been recorded from LNs (Flanagan and Mercer 1989; Christensen et al. 1993, 2001; Sun et al. 1993; Galizia and Kimmerle 2004; Krofczik et al. 2009). If these are not due to multiple neuron recordings or to gap-junctions between neurons, multiple 
spike heights might reflect distributed active membranes, resulting in multiple spike initiation zones. Whether heteroLNs are capable of performing both, elemental and configural odorant processing, in an odor-context-dependent manner, remains to be shown in future studies. If this hypothesis were confirmed, this could explain their high number in the honeybee AL. Honeybees rely to a greater degree on flexible odorant coding than most species, given that they are flower-constant polylectic pollen and nectar foragers. Thus, their olfactory system must be capable of processing and memorizing many odorant mixtures as unique flower identifiers. In addition, social insects have a complex communication system relying on many odors that function as pheromones, for which so far no dedicated subgroup of glomeruli forming a labeled line has been found. Thus, the same combinatorial logic may be used for floral odorants and for pheromones. In this situation, it is an advantage for the bee to identify both the odorant elements in a mixture, and the uniqueness of the mixture itself. The 'elemental' and 'configural' neurons presented here are likely to form an important role in the neural networks performing this task.

Acknowledgments We thank two anonymous referees for their constructive and helpful comments. We thank Martin P. Nawrot for providing data analysis scripts, practical advice and comments on the manuscript. We are grateful to David Gustav and Birgit Rapp for beekeeping, Jacob Stierle, Christoph Kleineidam, Henning Proske, Cyrille Girardin and Sabine Kreissl for fruitful discussions, Christine Dittrich and Tobias Müller for technical assistance, Jago Wallenschus and Corinna Geiss for identification of glomeruli and Marit Stranden for help with neuron reconstructions. This work was supported by the Landesgraduiertenföderung Baden-Würtemberg, the Deutsche Akademische Austausch Dienst, and the DFG.

Open Access This article is distributed under the terms of the Creative Commons Attribution Noncommercial License which permits any noncommercial use, distribution, and reproduction in any medium, provided the original author(s) and source are credited.

\section{References}

Ache BW, Young JM (2005) Olfaction: diverse species, conserved principles. Neuron 48(3):417-430. doi:10.1016/j.neuron.2005. 10.022

Andersson MN, Binyameen M, Sadek MM, Schlyter F (2011) Attraction modulated by spacing of pheromone components and anti-attractants in a bark beetle and a moth. J Chem Ecol 37(8):899-911. doi:10.1007/s10886-011-9995-3

Balderrama N, ñez JN, Giurfa M, Torrealba J, Albornoz ED, Almeida LO (1996) A deterrent response in honeybee (Apis mellifera) foragers: dependence on disturbance and season. J Insect Physiol 42:463-470

Balderrama N, nez JN, Guerrieri F, Giurfa M (2002) Different functions of two alarm substances in the honeybee. J Comp Physiol A Neuroethol Sens Neural Behav Physiol 188(6): 485-491. doi:10.1007/s00359-002-0321-y
Baraldi R, Rapparani F, Rossi F, Latella A, Ciccioli P (1999) Volatile organic compound emissions from flowers of the most occurring and economically important species of fruit trees. Phys Cem Earth 24:729-732

Bhandawat V, Olsen SR, Gouwens NW, Schlief ML, Wilson RI (2007) Sensory processing in the drosophila antennal lobe increases reliability and separability of ensemble odor representations. Nat Neurosci 10(11):1474-1482. doi:10.1038/nn1976

Chou YH, Spletter ML, Yaksi E, Leong JCS, Wilson RI, Luo L (2010) Diversity and wiring variability of olfactory local interneurons in the drosophila antennal lobe. Nat Neurosci. doi: 10.1038/nn.2489 (journal club 15.02.2010)

Christensen TA, Waldrop BR, Harrow ID, Hildebrand JG (1993) Local interneurons and information processing in the olfactory glomeruli of the moth Manduca sexta. J Comp Physiol [A] 173(4):385-399

Christensen TA, D’Alessandro G, Lega J, Hildebrand JG (2001) Morphometric modeling of olfactory circuits in the insect antennal lobe: I. Simulations of spiking local interneurons. Biosystems 61(2-3):143-153

Dacks AM, Reisenman CE, Paulk AC, Nighorn AJ (2010) Histamineimmunoreactive local neurons in the antennal lobes of the hymenoptera. J Comp Neurol 518(15):2917-2933. doi:10.1002/ cne. 22371

Deisig N, Lachnit H, Giurfa M, Hellstern F (2001) Configural olfactory learning in honeybees: negative and positive patterning discrimination. Learn Mem 8(2):70-78. doi:10.11011m.38301

Deisig N, Lachnit H, Giurfa M (2002) The effect of similarity between elemental stimuli and compounds in olfactory patterning discriminations. Learn Mem 9(3):112-121. doi:10.1101/ $\operatorname{lm} .41002$

Deisig N, Lachnit H, Sandoz JC, Lober K, Giurfa M (2003) A modified version of the unique cue theory accounts for olfactory compound processing in honeybees. Learn Mem 10(3):199-208. doi:10.1101/lm.55803

Deisig N, Giurfa M, Lachnit H, Sandoz JC (2006) Neural representation of olfactory mixtures in the honeybee antennal lobe. Eur J Neurosci 24(4):1161-1174. doi:10.1111/j.1460-9568.2006.04959.x

Deisig N, Giurfa M, Sandoz JC (2010) Antennal lobe processing increases separability of odor mixture representations in the honeybee. J Neurophysiol 103(4):2185-2194. doi:10.1152/jn. 00342.2009

Devaud JM, Masson C (1999) Dendritic pattern development of the honeybee antennal lobe neurons: a laser scanning confocal microscopic study. J Neurobiol 39(4):461-474

Distler PG, Gruber C, Boeckh J (1998) Synaptic connections between GABA-immunoreactive neurons and uniglomerular projection neurons within the antennal lobe of the cockroach, Periplaneta americana. Synapse 29(1):1-13

Flanagan D, Mercer AR (1989) Morphology and response characteristics of neurones in the deutocerebrum of the brain in the honeybee Apis mellifera. J Comp Physiol 164:483-494

Fonta C, Sun XJ, Masson C (1993) Morphology and spatial distribution of bee antennal lobe interneurones to odours. Chem Senses 18(2):101-119, doi:10.1093/chemse/18.2.101, http:// chemse.oxfordjournals.org/cgi/content/abstract/18/2/101, http:// chemse.oxfordjournals.org/cgi/reprint/18/2/101.pdf

Galizia CG, Menzel R (2001) The role of glomeruli in the neural representation of odours: results from optical recording studies. J Insect Physiol 47(2):115-130

Galizia CG (2008) Insect olfaction. In: The senses: a comprehensive reference. pp 725-769

Galizia CG, Kimmerle B (2004) Physiological and morphological characterization of honeybee olfactory neurons combining electrophysiology, calcium imaging and confocal microscopy. 
J Comp Physiol A Neuroethol Sens Neural Behav Physiol 190(1):21-38. doi:10.1007/s00359-003-0469-0

Galizia CG, Joerges J, Küttner A, Faber T, Menzel R (1997) A semiin-vivo preparation for optical recording of the insect brain. J Neurosci Methods 76(1):61-69

Galizia CG, McIlwrath SL, Menzel R (1999) A digital threedimensional atlas of the honeybee antennal lobe based on optical sections acquired by confocal microscopy. Cell Tissue Res 295(3):383-394

Gascuel J, Masson C (1991) A quantitative ultrastructural study of the honeybee antennal lobe. Tissue Cell 23(3):341-355

Junek S, Kludt E, Wolf F, Schild D (2010) Olfactory coding with patterns of response latencies. Neuron 67:872-884

Krofczik S, Menzel R, Nawrot MP (2009) Rapid odor processing in the honeybee antennal lobe network. Front Comput Neurosci 2:1-13

Lei H, Vickers N (2008) Central processing of natural odor mixtures in insects. J Chem Ecol 34(7):915-927. doi:10.1007/s10886008-9487-2

Linster C, Sachse S, Galizia CG (2005) Computational modeling suggests that response properties rather than spatial position determine connectivity between olfactory glomeruli. J Neurophysiol 93(6):3410-3417. doi:10.1152/jn.01285.2004

Malun D (1991) Inventory and distribution of synapses of identified uniglomerular projection neurons in the antennal lobe of Periplaneta americana. J Comp Neurol 305(2):348-360. doi: 10.1002/cne.903050215

Meier R, Egert U, Aertsen A, Nawrot MP (2008) FIND—a unified framework for neural data analysis. Neural Netw 21(8):10851093. doi:10.1016/j.neunet.2008.06.019

Menzel R, Hammer M, Müller U, Rosenboom H (1996) Behavioral, neural and cellular components underlying olfactory learning in the honeybee. J Physiol Paris 90(5-6):395-398

Mobbs PG (1982) The brain of the honeybee Apis mellifera I. the connections and spatial organization of the mushroom bodies. Philos Trans R Soc Lond B 298:309-354

Nawrot MP, Aertsen A, Rotter S (2003) Elimination of response latency variability in neuronal spike trains. Biol Cybern 88(5):321-334. doi:10.1007/s00422-002-0391-5

Ng M, Roorda RD, Lima SQ, Zemelman BV, Morcillo P, Miesenbck $G$ (2002) Transmission of olfactory information between three populations of neurons in the antennal lobe of the fly. Neuron 36(3):463-474

Olsen SR, Wilson RI (2008) Lateral presynaptic inhibition mediates gain control in an olfactory circuit. Nature 452(7190):956-960. doi:10.1038/nature06864

Omata A, Yomogida K, Nakamura S (1990) Volatile components of apple flowers. Flavour Fragr J 5:19-22

Pareto A (1972) Spatial distribution of sensory antennal fibres in the central nervous system of worker bees. Z Zellforsch Mikrosk Anat 131(1):109-140

Pouzat C, Chaffiol A (2009) Automatic spike train analysis and report generation. An implementation with $\mathrm{r}, \mathrm{r} 2 \mathrm{html}$ and star. J Neurosci Methods 181(1):119-144. doi:10.1016/j.jneumeth.2009.01.037
Pouzat C, Delescluse M, Viot P, Diebolt J (2004) Improved spikesorting by modeling firing statistics and burst-dependent spike amplitude attenuation: a markov chain monte carlo approach. J Neurophysiol 91(6):2910-2928. doi:10.1152/jn.00227.2003

Sachse S, Galizia CG (2003) The coding of odour-intensity in the honeybee antennal lobe: local computation optimizes odour representation. Eur J Neurosci 18(8):2119-2132

Sachse S, Peele P, Silbering AF, Ghmann M, Galizia CG (2006) Role of histamine as a putative inhibitory transmitter in the honeybee antennal lobe. Front Zool 3:22. doi:10.1186/1742-9994-3-22

Sato K, Touhara K (2009) Insect olfaction: receptors, signal transduction, and behavior. Results Probl Cell Differ 47:121-138

Savitzky A, Golay M (1964) Smoothing and differentiation of data by simplified least squares procedures. Anal Chem 36:1627-1639

Seki Y, Kanzaki R (2008) Comprehensive morphological identification and gaba immunocytochemistry of antennal lobe local interneurons in bombyx mori. J Comp Neurol 506(1):93-107. doi:10.1002/cne. 21528

Shepherd GM, Chen WR, Willhite D, Migliore M, Greer CA (2007) The olfactory granule cell: from classical enigma to central role in olfactory processing. Brain Res Rev 55(2):373-382. doi: 10.1016/j.brainresrev.2007.03.005

Silbering AF, Galizia CG (2007) Processing of odor mixtures in the drosophila antennal lobe reveals both global inhibition and glomerulus-specific interactions. J Neurosci 27(44):11,96611,977. doi:10.1523/JNEUROSCI.3099-07.2007

Silbering AF, Okada R, Ito K, Galizia CG (2008) Olfactory information processing in the drosophila antennal lobe: anything goes. J Neurosci 28(49):13075-13087. doi:10.1523/JNEUROSCI. 2973-08.2008

Stocker RF, Lienhard MC, Borst A, Fischbach KF (1990) Neuronal architecture of the antennal lobe in Drosophila melanogaster. Cell Tissue Res 262(1):9-34

Stopfer M, Jayaraman V, Laurent G (2003) Intensity versus identity coding in an olfactory system. Neuron 39(6):991-1004

Sun XJ, Fonta C, Masson C (1993) Odour quality processing by bee antennal lobe interneurones. Chem Senses 18(4):355-377. doi: 10.1093/chemse/18.4.355 (http://chemse.oxfordjournals.org/cgi/ content/abstract/18/4/355, http://chemse.oxfordjournals.org/cgi/ reprint/18/4/355.pdf)

Tabor R, Yaksi E, Weislogel JM, Friedrich RW (2004) Processing of odor mixtures in the zebrafish olfactory bulb. J Neurosci 24(29):6611-6620. doi:10.1523/JNEUROSCI.1834-04.2004

Tollsten L, Knudsen JT (1992) Scent in dioecious Salix (salicaceaae) - a cue determining the pollination system? Plant Syst Evol 182:229-237

Urban NN, Arevian AC (2009) Computing with dendrodendritic synapses in the olfactory bulb. Ann NY Acad Sci 1170:264-269. doi:10.1111/j.1749-6632.2009.03899.x

Vetter RS, Sage AE, Justus KA, Card RT, Galizia CG (2006) Temporal integrity of an airborne odor stimulus is greatly affected by physical aspects of the odor delivery system. Chem Senses 31(4):359-369. doi:10.1093/chemse/bjj040 